\title{
Generation of a Single UV Pulse from a near-IR Pulse Burst
}

\author{
Edgar Kaksis ${ }^{1}$, Ignas Astrauskas ${ }^{1}$, Tobias Flöry ${ }^{1}$, Giedrius Andriukaitis ${ }^{1}$, \\ Tadas Balčiūnas ${ }^{1}$, Audrius Pugžlys ${ }^{1}$, Andrius Baltuškaa ${ }^{1}$ and Tenio Popmintchev ${ }^{2}$
}

1. Photonics Institute, TU Wien, Gusshausstrasse 27 E-387, A-1040 Vienna, Austria

2. Department of Physics, Center of Advance Nanoscience, University of California, San Diego, 9500 Gilman Drive, La Jolla, California 92093, USA

High harmonic generation (HHG) requires high peak-power laser sources. Most of the well-known high peak power lasers are operating in the NIR wavelength region. Recently it was shown that the HHG photon flux and photon cut-off energy can be enhanced by multiple ionization when an intense UV driver pulse is used [1]. High average and high peak power UV sources operating around and below $250 \mathrm{~nm}$ are required for many other applications, such as ablation in ophthalmology, materials processing and photoelectron spectroscopy. Due to lack of ultrafast high peak power lasers operating in UV, generation of ultrashort UV pulses is possible by upconverting frequency of NIR laser. This is done by cascaded $4^{\text {th }}$ harmonic generation in nonlinear crystals with efficiency higher than $40 \%$ [2]. However, to obtain high pulse energies in UV region, high energy IR pump is necessary. This becomes increasingly difficult for femtosecond laser pulses because of the optical damage problem in CPA systems. Very high pulse stretching rates in a CPA become unfeasible due to the limited size of dispersive optics. Alternatively, the intensity in the laser cavity can be decreased by using a pulse burst which effectively increases the pulse duration. Therefore, this approach is also suitable for increasing energy throughput in fiber delivery and fiber post compression schemes [3].

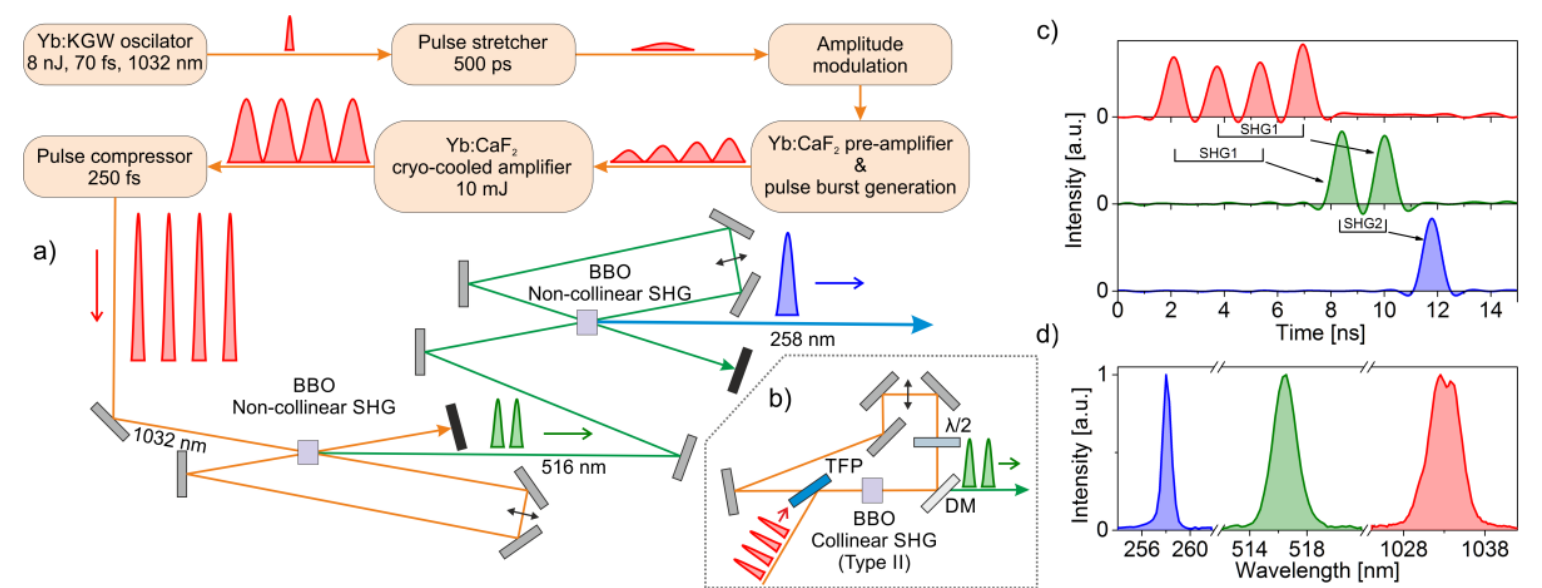

Fig. 1. Color coding: red - NIR pulses at $1032 \mathrm{~nm}$; green $-2^{\text {nd }}$ harmonic signal at $516 \mathrm{~nm}$; blue -4 th harmonic signal at $258 \mathrm{~nm}$. a) Schematic setup b) layout for type II collinear generation; c) measured and deconvoluted pulses d) measured corresponding pulse spectra of the fundamental-frequency pulses and the $2^{\text {nd }}$ and $4^{\text {th }}$ harmonics;

In this contribution we propose and experimentally test the scheme for converting a burst of 4 pulses into a single $4^{\text {th }}$ harmonic pulse (Fig. 1a). The scheme is extendable and can be used for $5^{\text {th }}$ harmonic generation from a 5 -pulse train. The pulse train is generated in a regenerative pre-amplifier by populating it with several successive pulses from the seed oscillator. The burst is further amplified to multi $\mathrm{mJ}$ level in a cryogenically cooled $\mathrm{Yb}: \mathrm{CaF}_{2}$ RA and compressed to $\sim 250 \mathrm{fs}$. The pulse burst is then launched into the first stacking loop, which by non-collinear (type I) sum-frequency generation in a $1.2 \mathrm{~mm}$ long BBO crystal combines the 4-pulse burst into a 2-pulse burst at twice the frequency. The Second stacking loop $(0.5 \mathrm{~mm} \mathrm{BBO})$ converts the pulse burst into a single intense pulse at the $4^{\text {th }}$ harmonic (UV) of the driving laser. This pulse stacking approach, in comparison to interferometric stacking schemes [4], does not require active phase stabilization. Efficient generation in non-collinear configuration for bigger beam sizes might need pulse front tilting, therefore, we suggest a configuration of the stacking loop using collinear (type II) sum-frequency generation (Fig. 1b). Measured pulses in the time domain are shown in Fig. 1c while Fig. 1d depicts the corresponding spectra.

The work is underway to increase UV pulse energy by boosting the pulse burst to hundreds of $\mathrm{mJ}$ in our multipass amplifier and by adding one more pulse to the burst to generate the $5^{\text {th }}$ harmonic in an additional sum frequency generation stage.

\section{References}

[1] D. Popmintchev et al., Science, 350(6265), 1225-1231 (2015).

[2] D. Bruneau, A. Tournade, and E. Fabre, Appl. Opt., 24(22), 3740-3745 (1985).

[3] H. Jacqmin, A. Jullien, B. Mercier, and R. Lopez-Martens, J. Opt. Soc. Am. B., 32(9), 1901-1909 (2015).

[4] T. Zhou, J. Ruppe, C. Zhu, I-N. Hu, J. Nees, and A. Galvanauskas, Opt. Exp., 23(6), 7442-7462 (2015).

(C) 2017 IEEE. Personal use of this material is permitted. Permission from IEEE must be obtained for all other uses, in any current or future media, including reprinting/republishing this material for advertising or promotional purposes, creating new collective works, for resale or redistribution to servers or lists, or reuse of any copyrighted component of this work in other works. 